\title{
Finite element analysis of mechanical stress of the hip joint in patients with posterior pelvic inclination
}

\author{
DAISUKE SAKUMA $^{1,2}$, YASUHIRO ISHIDOU ${ }^{1}$, YUSUKE FUJIMOTO ${ }^{1,2}$, SHUNSUKE NAKAMURA $^{2}$, \\ TOSHIRO IJUIN $^{2}$, SATOSHI NAGANO ${ }^{3}$ and NOBORU TANIGUCHI ${ }^{1,2}$
}

\begin{abstract}
Departments of ${ }^{1}$ Medical Joint Materials, and ${ }^{2}$ Orthopaedic Surgery, Graduate School of Medical and Dental Sciences, Kagoshima University, Kagoshima 890-8520; ${ }^{3}$ Department of Clinical Physical Therapy, School of Health Sciences, Faculty of Medicine, Kagoshima University, Kagoshima 890-8544, Japan
\end{abstract}

Received January 12, 2022; Accepted February 18, 2022

DOI: 10.3892/wasj.2022.145

\begin{abstract}
Abnormal mechanical loading is a main external factor affecting the development of osteoarthritis (OA). Excessive posterior pelvic inclination may decrease the loading area of the acetabulum, increasing mechanical stress to the articular surface and resulting in hip OA. However, exactly how much posterior pelvic inclination produces an excessive load on the articular surface remains unknown. The present study investigated the mechanical stress of 27 hips in 19 women [unilateral (right or left) hip joints of 11 women and bilateral (right and left) hip joints of 8 women were analyzed] with or without acetabular dysplasia by finite element analysis. Patient-specific finite element models were constructed from computed tomography data obtained in the supine position. The posterior pelvic inclination in the models was changed from 0 to 30 degrees in five-degree increments. The association between equivalent stress in the hip joint and the pelvic inclination or acetabular dysplasia was analyzed. The equivalent stress for the femoral head in the original position was $0.97 \mathrm{MPa}(0.91-1.01)$ in normal hip joints and $1.18 \mathrm{MPa}$ (1.00-1.28) in hips with acetabular dysplasia $(\mathrm{P}=0.023)$. The equivalent stress significantly increased at $>25$ degrees of posterior inclination. In normal hips, when the pelvic posterior inclination was increased by 25 and 30 degrees, the equivalent stress was 1.21 (1.11-1.35) and 1.24 (1.20-1.47) MPa, respectively $(\mathrm{P}=0.029$ and 0.010 , respectively). The mechanical stress of normal hip joints serially increased as the posterior
\end{abstract}

Correspondence to: Professor Satoshi Nagano, Department of Clinical Physical Therapy, School of Health Sciences, Faculty of Medicine, Kagoshima University, 8-35-1 Sakuragaoka, Kagoshima 890-8544, Japan

E-mail: naga@m2.kufm.kagoshima-u.ac.jp

Abbreviations: OA, osteoarthritis; FE, finite element; CT, computed tomography

Key words: finite element analysis, mechanical stress, posterior pelvic inclination, hip osteoarthritis pelvic inclination increased, reaching a level almost equivalent to that of hip joints with acetabular dysplasia at 25 degrees of posterior inclination. On the whole, the present study demonstrates that posterior pelvic inclination may be a mechanical factor affecting the development of OA in patients without acetabular dysplasia, particularly when the posterior inclination exceeds 25 degrees.

\section{Introduction}

Abnormal mechanical loading is a main external factor affecting the development of osteoarthritis (OA). Physiological loading plays a critical role in the maintenance of the cartilage matrix. However, excessive loading causes an imbalance between the degradation and synthesis of matrix macromolecules, thus resulting in OA $(1,2)$. Obesity and a heavy physical workload influence the occurrence of hip OA $(3,4)$. Acetabular dysplasia is an anatomical factor that increases the mechanical stress on the articular surface of the hip joint $(5,6)$. However, the incidence of OA with concurrent acetabular dysplasia is lower in patients of an advanced age $(7,8)$.

Age-related spinal degenerative change commonly results in spinal kyphosis in older individuals. Patients with spinal kyphosis develop posterior pelvic inclination to compensate for their sagittal spinal imbalance (9-11). Excessive posterior pelvic inclination may uncover the anterior acetabulum (12). The decrease in the loading area of the femoral head presumably increases mechanical stress to the articular surface, resulting in OA. To date, only a limited number of studies have described such a mechanism in the etiology of hip OA $(13,14)$. Kitamura et al (15) recently analyzed the equivalent stress of the acetabular surface at three pelvic tilt positions relative to the functional pelvic plane $\left(10^{\circ}\right.$ anterior tilt, no tilt, and $10^{\circ}$ posterior tilt). They demonstrated that the mean contact area was smaller and that the mean maximum contact pressure and median maximum equivalent stress were larger in anteriorly than posteriorly tilted pelvises. However, exactly how much posterior pelvic inclination produces an excessive load on the articular surface and is a risk factor for OA onset remains unknown.

The present study was thus performed to determine the amount of posterior pelvic inclination required to reproduce the loading condition of the dysplastic hip joints using finite 
element (FE) analysis. To this end, the mechanical stress of the articular surface of the hip was analyzed while posterior pelvic inclination was serially increased in five-degree increments. In addition, the mechanical stress was compared between normal hips and hips with acetabular dysplasia with or without posterior pelvic inclination.

\section{Patients and methods}

Patients. Patients treated for coxalgia at Kagoshima University Medical Hospital from January, 2011 to December, 2016 were retrospectively evaluated. The criteria for enrollment in the present study were as follows: A hip joint space of $>2.0 \mathrm{~mm}$, no radiographic evidence of formation of osteophytes or cysts or severe sclerotic change, deformity of the femoral head to eliminate secondary changes in osteoarthritic progression, and no previous operation on the hip joint. The present study finally included 27 hips of 19 women [unilateral (right or left) hip joints of 11 women and bilateral (right and left) hip joints of 8 women were analyzed]. All data were retrospectively collected from medical records (Table I). The research protocol of the present study was approved by the Institutional Ethics Committee on Clinical Research of Kagoshima University, and the study was performed in compliance with the Declaration of Helsinki. Patient consent was waived by the committee as this study was a retrospective observational study.

Radiographic measurements. The Sharp's angle of the hip and pelvic inclination were measured on frontal X-ray images of the bilateral hip joints. A Sharp's angle of $\geq 45$ degrees was defined as acetabular dysplasia in the present study. The pelvic inclination was calculated based on measurements from anteroposterior pelvic radiographs according to a previous report (16). In summary, the pelvic inclination was calculated using the following equation: $\mathrm{A}=-69.0^{\circ} \times \mathrm{L} / \mathrm{T}+61.6^{\circ}$ in women, where $\mathrm{L} / \mathrm{T}$ is the longitudinal/transverse axis length ratio of the pelvic cavity on the anteroposterior pelvic radiograph. The equation was produced by comparing the pelvic inclination with the L/T ratio using Japanese pelvic bones. According to an original study using anteroposterior pelvic radiographs of normal hip joints, the standard pelvic inclination was $19.97 \pm 5.93^{\circ}$ in women (16)

FE analysis. Femoral and pelvic models were created based on computed tomography (CT) scans with a slice thickness of $3 \mathrm{~mm}$. CT imaging was performed in the supine position. This position was defined as the original position.

FE models of the femur and pelvis were generated from the CT data using Mechanical Finder version 9.0 software (Research Centre for Computational Mechanics Inc.). This software constructs FE models demonstrating the shape of the individual bone and the distribution of its density; it also implements the FE method for solving linear algebraic equations using the displacement method (17). FE mesh models were produced using ANSYS ICEM CFD version 18.0 (ANSYS Inc.) with this software. FE models of the femur were produced using the methods described in the study by Bessho et al (17) and their methods were applied to the pelvis. Image processing was performed using Mechanical Finder version 10.0 (Research Centre for Computational Mechanics Inc., Tokyo, Japan).

Subsequently, three-dimensional FE models were constructed for each patient (Fig. 1). A mesh size of $<2 \mathrm{~mm}$ could not be solved due to the limited capacity of the computer used. A total of five models of different mesh sizes were created to perform the mesh sensitivity test: 2.0 to $4.0 \mathrm{~mm}$, 2.5 to $5.0 \mathrm{~mm}, 3.0$ to $6.0 \mathrm{~mm}, 3.5$ to $7.0 \mathrm{~mm}$ and 4.0 to $8.0 \mathrm{~mm}$. The mesh convergence test used in the present study calculated the equivalent stress. The percentage change of the equivalent stress between the 2.0 - to $4.0-\mathrm{mm}$ model and the 2.5 - to $5.0-\mathrm{mm}$ model was $0.70 \%$. The percentage changes between the 2.0- to $4.0-\mathrm{mm}$ and 3.0- to 6.0-mm models, 2.0 to $4.0-\mathrm{mm}$ and $3.5-$ to $7.0-\mathrm{mm}$ models, and $2.0-$ to $4.0-\mathrm{mm}$ and 4.0 - to $8.0-\mathrm{mm}$ models were $1.13,3.70$ and $5.02 \%$, respectively. Therefore, the $2.0-$ to $4.0-\mathrm{mm}$ mesh size was used. These models had 2.0- to 4.0-mm tetrahedral elements for the inner cortical and cancellous bone and three-nodal point shell elements with a thickness of $0.4 \mathrm{~mm}$ for the outer cortical bone. Articular cartilage was modeled as a homogeneous isotropic material (18). The articular cartilage of the femoral head was presumed to be spherical and to have a mean thickness of $2.0 \mathrm{~mm}$ (19). The models of articular cartilage had 0.5 - to $1.0-\mathrm{mm}$ tetrahedral elements. FE models of the pelvis were constructed with $\sim 300,000$ elements, and those of the femur were constructed with $\sim 130,000$ elements. The elastic modulus of the cortical and cancellous bone was determined from CT density values using the equations proposed in the study by Keyak et al (20) (Table II). Poisson's ratio of the cortical and cancellous bone was $0.40(20,21)$. Articular cartilage had an elastic modulus of 10.35 MPa and a Poisson's ratio of 0.40 (18). The models presumed completely bonded interfaces between cartilage and bone, while the coefficient of friction between cartilages was presumed to be 0 (Fig. 1). The applied loading condition was based on the study by Ike et al (18). A load of $1,600 \mathrm{~N}$ was applied vertically to the sacral spine. The models were presumed to represent the patient standing on one leg. Pauwels (22) previously reported that the contact force of the femoral head was three times the body weight when standing on one leg. The mean body weight of Japanese women was $\sim 53 \mathrm{~kg}$ in 2015 . Therefore, the load was defined as 1,600 N. Of note, two boundary conditions were used in the present study: The distal parts of the femoral shafts were fully restrained, and the median line of the pelvis was fully restrained except for Z-axial displacement (Fig. 2). In each model, three-dimensional FE models were also constructed by changing the pelvic inclination from the original position to +30 degrees in five-degree increments.

The Drucker-Prager yield criterion was used. The mean equivalent stress in the subchondral bone was used. The mean equivalent stress in the part of the femoral head covered by the acetabular roof was measured.

Statistical analysis. Age, Sharp's angle of the hip and pelvic inclination values are expressed as the mean \pm standard deviation. The equivalent stress is expressed as the median and interquartile range. The Kolmogorov-Smirnov and Shapiro-Wilk tests were used to evaluate the data. The equivalent stress did not follow a normal distribution; therefore, the Mann-Whitney U test was used to compare the equivalent 
Table I. Baseline characteristics of the patients in the present study.

\begin{tabular}{llcc}
\hline & $\begin{array}{c}\text { Normal } \\
(\mathrm{n}=16 \text { hips })\end{array}$ & $\begin{array}{c}\text { Acetabular dysplasia } \\
(\mathrm{n}=11 \text { hips })\end{array}$ & $\begin{array}{c}\text { P-value } \\
\text { Age, years }\end{array}$ \\
Sharp's angle of the hip & $59.0 \pm 19.7$ & $46.0 \pm 13.5$ & 0.98 \\
Pelvic inclination & $39.3 \pm 3.3$ & $47.6 \pm 2.1$ & $<0.001^{\text {a }}$ \\
\hline
\end{tabular}

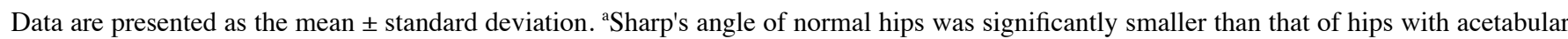
dysplasia.

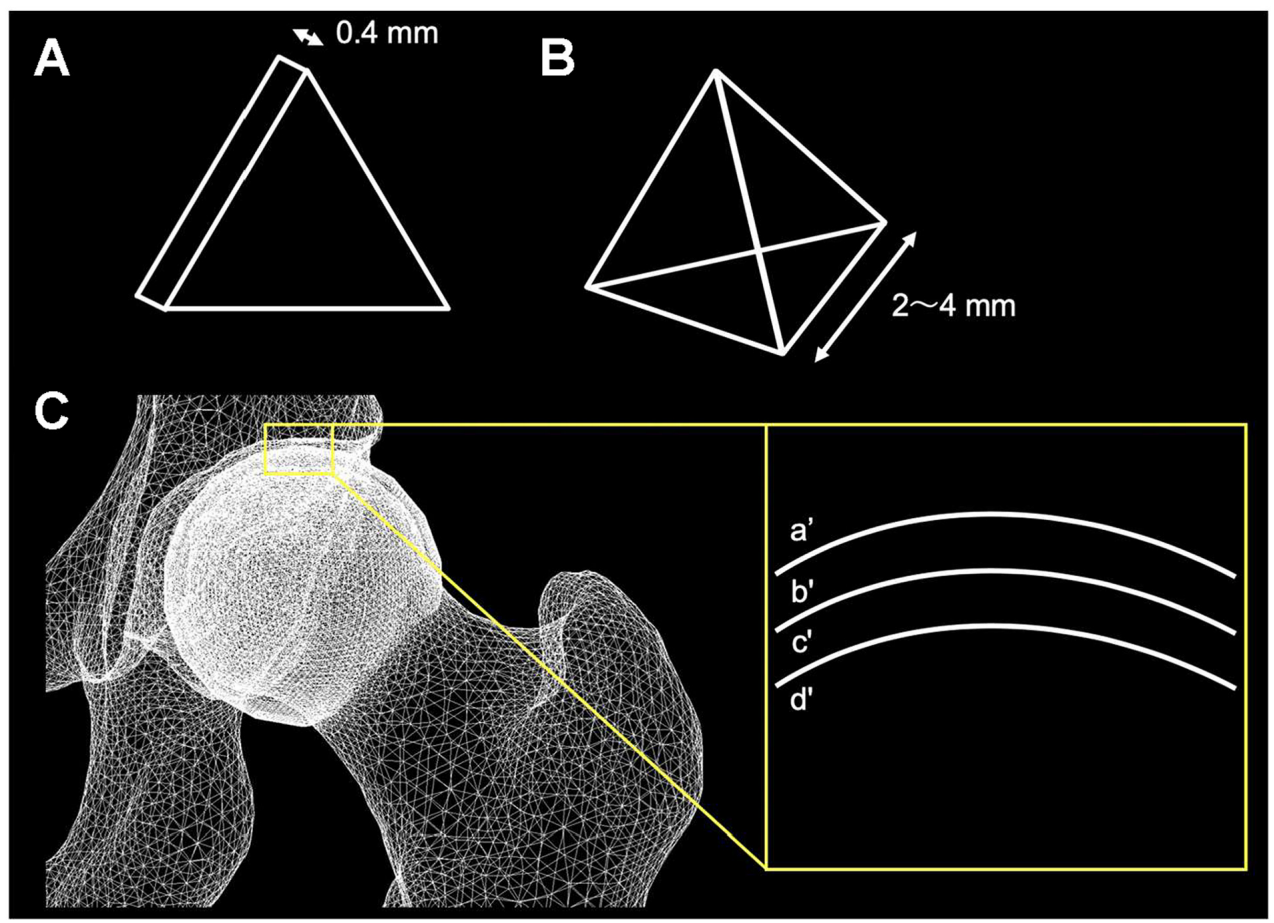

Figure 1. Details of finite element meshes. Linear elements were used. (A) Shell element with a thickness of 0.4 mm. (B) 2- to 4-mm tetrahedral elements. (C) Three-dimensional FE model and the contact model and settings. (a') Bone of the acetabular side. (b') Cartilage of the acetabular side. (c') Cartilage of the femoral head side. (d') Bone of the femoral head side.

stress of the femoral head between normal hip joints and hips affected by acetabular dysplasia. A value of $\mathrm{P}<0.05$ was considered to indicate a statistically significant difference. The equivalent stress measured for each pelvic inclination was compared. The Kruskal-Wallis test was used to compare the equivalent stress among the different pelvic inclinations. If the difference was significant, the Bonferroni correction was used. All statistical analyses were performed using EZR version 1.35 (Saitama Medical Center, Jichi Medical University, Saitama, Japan), which is a graphical user interface for R (The $\mathrm{R}$ Foundation for Statistical Computing). More precisely, it is a modified version of $\mathrm{R}$ commander designed to add statistical functions frequently used in biostatistics (23).

\section{Results}

FE analysis was used to compare normal hips with hips affected by acetabular dysplasia, changing the pelvic inclination from the original position to 30 degrees. The equivalent stress for the femoral head at the original position was $0.97 \mathrm{MPa}(0.91-1.01)$ in normal hip joints and 1.18 MPa (1.00-1.28) in hips affected by acetabular dysplasia ( $\mathrm{P}=0.023$; Fig. 3 ).

The distribution and volume of the equivalent stress to the femoral head at the original position and with 30 degrees of posterior inclination is presented in Fig. 3. In hips with acetabular dysplasia, the equivalent stress one-sidedly increased at the anterior portion of the femoral head compared with the normal hip (Fig. 4A and C). In both normal hips and hips with acetabular dysplasia, the equivalent stress was markedly increased at 30 degrees of posterior inclination (Fig. 4B and D).

The equivalent stress of the femoral heads under various conditions in which the pelvic inclination was changed from the original position to 30 degrees in 5-degree increments is shown in Table III. In both normal hips and hips with acetabular dysplasia, the equivalent stress increased in a stepwise manner. In the normal hips, when the pelvic posterior inclination was increased by 25 and 30 degrees, the equivalent 
Table II. Equations used to calculate the elastic modulus of bone.

\begin{tabular}{lc}
\hline Bone density & Elastic modulus \\
\hline$\rho=0$ & 0.001 \\
$0<\rho \leq 0.27$ & $33,900 \mathrm{p}^{2.20}$ \\
$0.27<\rho<0.6$ & $5307 \mathrm{p}+469$ \\
$0.6 \leq \rho$ & $10,200 \mathrm{p}^{2.01}$ \\
\hline
\end{tabular}

The information in the table is derived from a previous study (20). ' $\rho$ ' represents the density of the bone; $\mathrm{p}\left(\mathrm{g} / \mathrm{cm}^{3}\right)=(\mathrm{HU}+1.4246) \mathrm{x} 0.001 / 1.058$ $(\mathrm{HU}>-1)=0.0$ (HU $\leq-1)$, where HU indicates Hounsfield units.

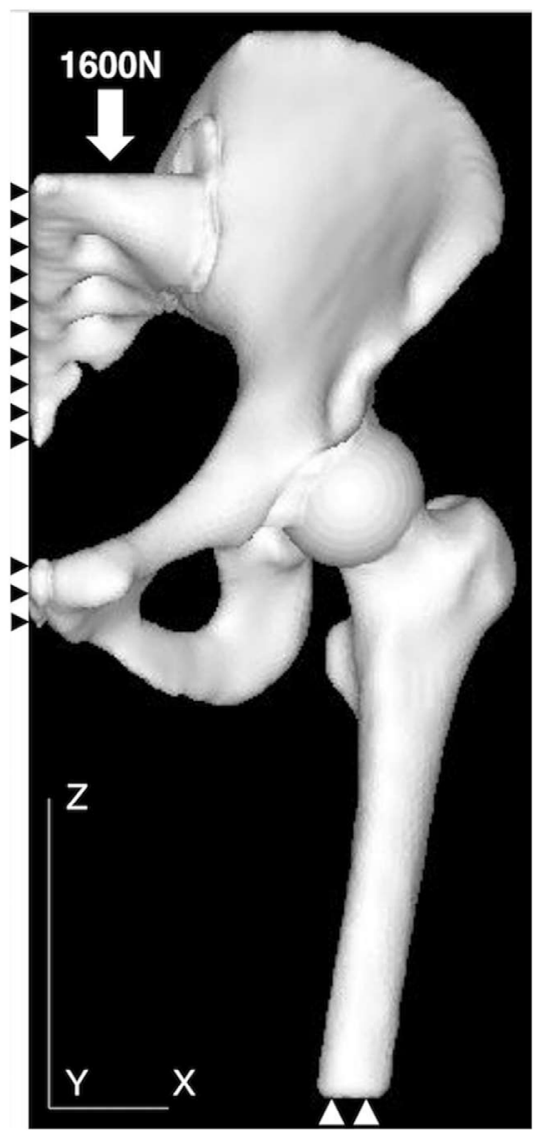

Figure 2. Loading and restriction conditions. A load of 1,600 N was applied vertically to the sacral spine (white arrow). The distal portion of the femoral shaft was fully restrained (white arrowheads). The median line of the pelvis was fully restrained except in Z-axial displacement (black arrowheads).

stress was $1.21(1.11=1.35)$ and $1.24(1.20=1.47) \mathrm{MPa}$, respectively $(\mathrm{P}=0.029$ and 0.010 , respectively). In the hips affected by acetabular dysplasia, when the posterior pelvic inclination was increased by 30 degrees, the equivalent stress was 1.61 (1.33-1.85) ( $\mathrm{P}=0.016$; Table III).

The equivalent stress of the femoral heads in patients with acetabular dysplasia was 1.22 -fold greater than that in patients with normal hip joints. The equivalent stress of the normal hip joint with 25 degrees of posterior pelvic inclination was 1.25 -fold greater than that of the original position. This overloading of the normal hip joint in patients

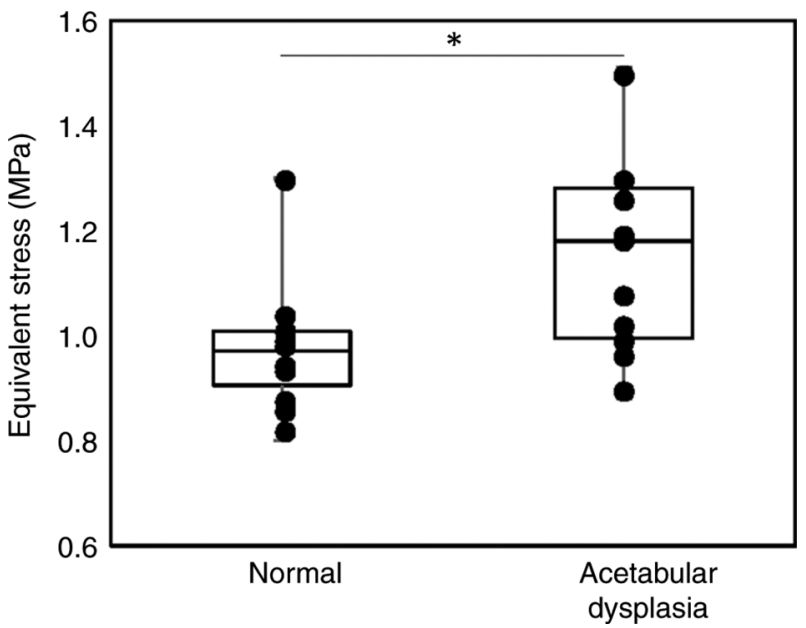

Figure 3. Equivalent stress in femoral head of normal hip or hip with acetabular dysplasia. The equivalent stress between the femoral head of the normal hip and the hip with acetabular dysplasia differed significantly ( $\left.{ }^{*} \mathrm{P}=0.023\right)$. The Mann-Whitney U test was used to analyze the data.

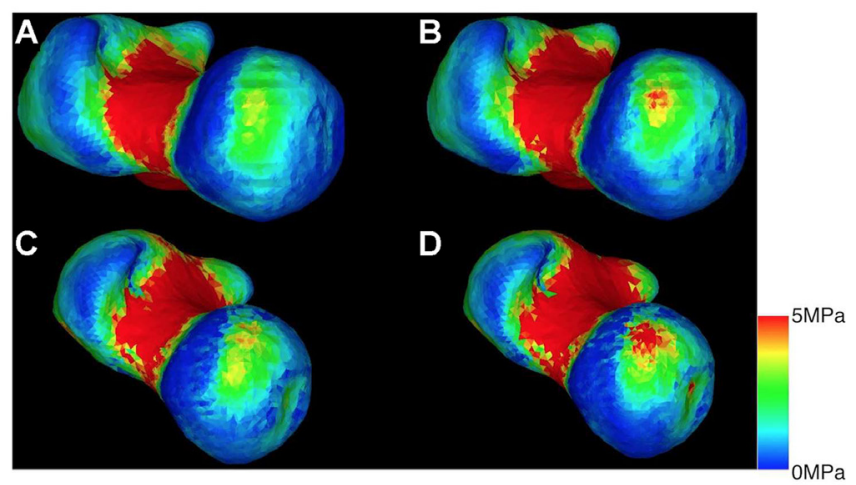

Figure 4. Diagram of equivalent stress. (A) Normal hip in the original position. (B) Normal hip with an additional 30 degrees. (C) Hip with acetabular dysplasia in the original position. (D) Hip with acetabular dysplasia with an additional 30 degrees.

with posterior pelvic inclination was the same as that in patients with acetabular dysplasia in the original position. Furthermore, the equivalent stress of the hip affected by acetabular dysplasia with 25 degrees of posterior pelvic inclination was 1.55 -fold greater than that of the normal hip at the original position (Fig. 5).

\section{Discussion}

The present study investigated the mechanical stress of hip joints with or without acetabular dysplasia by FE analysis. The mechanical stress when the posterior pelvic inclination changed from 0 to 30 degrees was also investigated.

A previous epidemiological study demonstrated that the population with acetabular dysplasia had a 4.3-fold higher risk of developing radiographic OA of the hip than the population without acetabular dysplasia (6). Excessive contact stress of the articular surface of the hip in patients with acetabular dysplasia predicts the onset of OA; therefore, various osteotomies have been designed to reduce the mechanical stress and risk of OA development (24). 
Table III. Equivalent stress in the femoral head in the patients in the present study.

A, Normal hip

Pelvic inclination angle

Median

Interquartile range

$\mathrm{P}$-value

Original position

0.97

0.91-1.01

$+5^{\circ}$

1.00

0.93-1.05

1.0

$+10^{\circ}$

1.02

0.93-1.10

1.0

$+15^{\circ}$

1.10

$0.99-1.16$

0.97

$+20^{\circ}$

1.16

1.05-1.24

0.11

$+25^{\circ}$

1.21

1.11-1.35

$0.029^{\mathrm{a}}$

$+30^{\circ}$

1.24

1.20-1.47

$0.010^{\mathrm{a}}$

B, Acetabular dysplasia

P-value

Pelvic inclination angle

Median

Interquartile range

(vs. original position)

\begin{tabular}{ll}
\hline Original position & 1.18 \\
$+5^{\circ}$ & 1.19 \\
$+10^{\circ}$ & 1.23 \\
$+15^{\circ}$ & 1.30 \\
$+20^{\circ}$ & 1.39 \\
$+25^{\circ}$ & 1.51 \\
$+30^{\circ}$ & 1.61
\end{tabular}

$1.00-1.28$
$1.05-1.31$
$1.08-1.37$
$1.12-1.40$
$1.20-1.48$
$1.26-1.67$
$1.33-1.85$

1.0

1.0

1.0

1.0

1.0

0.18

$\begin{array}{ll}.61 & 1.33-1.85\end{array}$

$0.016^{\mathrm{a}}$

The results are presented as the median with interquartile range. The unit of equivalent stress is MPa. The Kruskal-Wallis test was used for multiple comparisons. If statistical significance was found, the Bonferroni correction was used. 'Indicates a statistically significant difference vs. the original position.

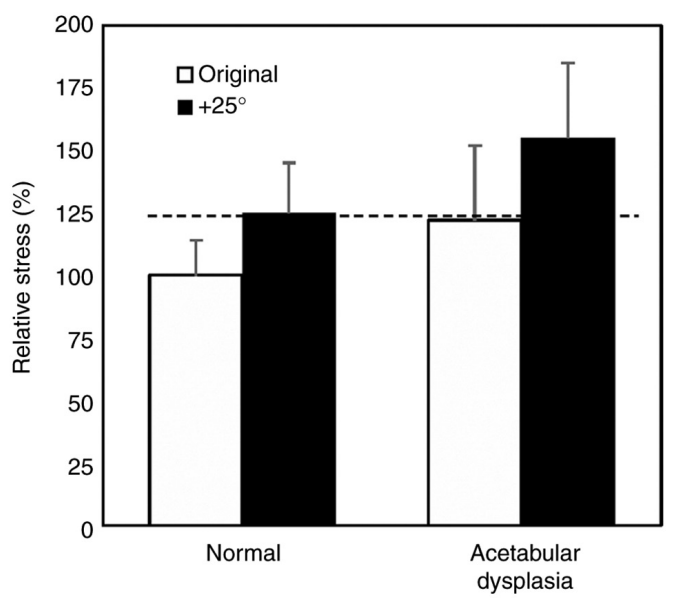

Figure 5. Equivalent stress under different conditions. The equivalent stress of the femoral heads with acetabular dysplasia was 1.22 -fold greater than that of normal hip joints. The equivalent stress of the normal hip joint with 25 degrees of posterior pelvic inclination was 1.25 -fold greater than that of the original position. Thus, overloading of normal hip joints with posterior pelvic inclination of 25 degrees was equivalent to that in hips with acetabular dysplasia in the original position (dashed line).

Patient-specific FE analysis has revealed that rotational acetabular osteotomy reduces the mechanical stress in the hip joint. The equivalent stress in the femoral head has been shown to decrease from 3.4 MPa (1.2-6.0) to 2.9 MPa (1.7-4.0) (18). Therefore, the post-operative equivalent stress in the femoral heads with acetabular dysplasia was 0.85 -fold greater than that in the pre-operative hips (18).

Hip-spine syndrome is a widely accepted morbid association between lumbar spine arthrosis and hip arthrosis (25). In patients of an advanced age, posterior pelvic inclination commonly increases due to various factors, including spinal kyphosis, paravertebral muscle weakness and failure of the pelvic support mechanism (26). Posterior pelvic inclination in patients with decreased lumbar lordosis reduces the femoral head area covered by the acetabulum (12). The present study demonstrated that normal hip joints without acetabular dysplasia had a mechanical stress almost equivalent to that in hip joints with acetabular dysplasia when posterior pelvic inclination was increased to 25 degrees (Fig. 5). Yoshimoto et al (27) analyzed several hip-spine alignment parameters, including pelvic tilt in patients with or without hip dysplasia. They found that patients who had hip OA without acetabular dysplasia tended to have decreased lumbar lordosis and increased posterior pelvic tilt, which was frequently observed in the older patients (27). With aging, posterior pelvic tilt increases as lumbar spinal degeneration decreases lumbar lordosis, resulting in secondary hip OA (spine-hip syndrome). Based on these current findings, posterior pelvic inclination of $>25$ degrees may be a risk factor for onset of OA secondary to pelvic malalignment, including hip-spine (spine-hip) syndrome. 
The present study has several limitations. First, the stress was analyzed for a single-legged stance. The stress of the hip joint during some activities (walking, running and stair climbing) could not be evaluated. Second, the bone models were created based on the CT images of each patient, but with the same loading conditions. Therefore, the results of the present study were subject to minor differences. Finally, the main limitation of the present study was the lack of direct epidemiological data showing that posterior pelvic inclination is a risk factor for OA. Such an epidemiological study may demonstrate the actual critical degree of posterior pelvic inclination that leads to hip OA.

In conclusion, the present study demonstrated that the mechanical stress of normal hip joints serially increased as posterior pelvic inclination increased, reaching a level almost equivalent to that of hip joints with acetabular dysplasia at 25 degrees of posterior pelvic inclination. The data presented herein suggest that posterior pelvic inclination may be a mechanical factor that affects the development of OA in patients without acetabular dysplasia, particularly when the posterior pelvic inclination exceeds 25 degrees.

\section{Acknowledgements}

Not applicable.

\section{Funding}

No funding was received.

\section{Availability of data and materials}

The datasets used and analyzed during the current study are available from the corresponding author on reasonable request.

\section{Authors' contributions}

DS and YI designed the study. DS, YF, SNak and TI performed data collection. DS, YI, SNag and NT analyzed the data and wrote the manuscript. SNak and NT revised the manuscript critically for important intellectual content. DS and SNag confirm the authenticity of all the raw data. All authors have read and approved the final manuscript.

\section{Ethics approval and consent to participate}

The present study was approved by the Ethics Committee on Clinical Research at Kagoshima University (no. 29-37), and the study was performed in compliance with the Declaration of Helsinki. Patient consent was waived by the committee as this study was a retrospective observational study.

\section{Patient consent for publication}

Not applicable.

\section{Competing interests}

The authors declare that they have no competing interests.

\section{References}

1. Sun HB: Mechanical loading, cartilage degradation, and arthritis. Ann N Y Acad Sci 1211: 37-50, 2010.

2. Chen C, Tambe DT, Deng L and Yang L: Biomechanical properties and mechanobiology of the articular chondrocyte. Am J Physiol Cell Physiol 305: 1202-1208, 2013.

3. Lievense AM, Bierma-Zeinstra SM, Verhagen AP, van Baar M, Verhaar JA and Koes BW: Influence of obesity on the development of osteoarthritis of the hip: A systematic review. Rheumatology (Oxford) 41: 1155-1162, 2002.

4. Lievense A, Bierma-Zeinstra S, Verhagen A, Verhaar J and Koes B: Influence of work on the development of osteoarthritis of the hip: A systematic review. J Rheumatol 28: 2520-2528, 2001.

5. Weinstein SL: Natural history of congenital hip dislocation $(\mathrm{CDH})$ and hip dysplasia. Clin Orthop Relat Res: 62-76, 1987.

6. Reijman M, Hazes JM, Pols HA, Koes BW and Bierma-Zeinstra SM: Acetabular dysplasia predicts incident osteoarthritis of the hip: The Rotterdam study. Arthritis Rheum 52: 787-793, 2005.

7. Ishidou Y, Matsuyama K, Sakuma D, Setoguchi T, Nagano S, Kawamura I, Maeda S and Komiya S: Osteoarthritis of the hip joint in elderly patients is most commonly atrophic, with low parameters of acetabular dysplasia and possible involvement of osteoporosis. Arch Osteoporos 12: 30, 2017.

8. Lane NE, Nevitt MC, Cooper C, Pressman A, Gore R and Hochberg M: Acetabular dysplasia and osteoarthritis of the hip in elderly white women. Ann Rheum Dis 56: 627-630, 1997.

9. Ailon T, Shaffrey CI, Lenke LG, Harrop JS and Smith JS: Progressive spinal kyphosis in the aging population. Neurosurgery 77 (Suppl 4): S164-S172, 2015.

10. Kasukawa Y, Miyakoshi N, Hongo M, Ishikawa Y, Kudo D, Suzuki M, Mizutani T, Kimura R, Ono Y and Shimada Y: Age-related changes in muscle strength and spinal kyphosis angles in an elderly Japanese population. Clin Interv Aging 12: 413-420, 2017.

11. Barrey C, Roussouly P, Le Huec JC, D'Acunzi G and Perrin G: Compensatory mechanisms contributing to keep the sagittal balance of the spine. Eur Spine J 22 (Suppl 6): S834-S841, 2013.

12. Watanabe W, Sato K, Itoi E, Yang K and Watanabe H: Posterior pelvic tilt in patients with decreased lumbar lordosis decreases acetabular femoral head covering. Orthopedics 25: 321-324, 2002.

13. Russell ME, Shivanna KH, Grosland NM and Pedersen DR: Cartilage contact pressure elevations in dysplastic hips: A chronic overload model. J Orthop Surg Res 1: 6, 2006.

14. Chegini S, Beck M and Ferguson SJ: The effects of impingement and dysplasia on stress distributions in the hip joint during sitting and walking: A finite element analysis. J Orthop Res 27: 295-201, 2009.

15. Kitamura K, Fujii M, Utsunomiya T, Iwamoto M, Ikemura S, Hamai S, Motomura G, Todo M and Nakashima Y: Effect of sagittal pelvic tilt on joint stress distribution in hip dysplasia: A finite element analysis. Clin Biomech (Bristol, Avon) 74: 34-41, 2020.

16. Doiguchi Y, Iwasaki K and Yamada K: Correlation between pelvic inclination and radiological shape of the pelvic cavity. Orthop Traumatol 41: 641-645, 1992.

17. Bessho M, Ohnishi I, Matsuyama J, Matsumoto T, Imai K and Nakamura K: Prediction of strength and strain of the proximal femur by a CT-based finite element method. J Biomech 40: $1745-1753,2007$

18. Ike H, Inaba Y, Kobayashi N, Yukizawa Y, Hirat Y, Tomioka M and Saito T: Effects of rotational acetabular osteotomy on the mechanical stress within the hip joint in patients with developmental dysplasia of the hip: A Subject-Specific finite element analysis. Bone Joint J 97-B: 492-497, 2015.

19. Zhao X, Chosa E, Totoribe K and Deng G: Effect of periacetabular osteotomy for acetabular dysplasia clarified by three-dimensional finite element analysis. J Orthop Sci 15: 632-640, 2010.

20. Keyak JH, Rossi SA, Jones KA and Skinner HB: Prediction of femoral fracture load using automated finite element modeling. J Biomech 31: 125-133, 1998.

21. Reilly DT and Burstein AH: The elastic and ultimate properties of compact bone tissue. J Biomech 8: 393-405, 1975.

22. Pauwels F: Biomechanics of the Normal and Diseased Hip. Theoretical Foundation, Technique and Results of Treatment. An Atlas. Springer-Verlag, 1976. 
23. Kanda Y: Investigation of the freely available easy-to-use software 'EZR' for medical statistics. Bone Marrow Transplant 48 452-458, 2013

24. Mavčič B, Iglič A, Kralj-Iglič V, Brand RA and Vengust R: Cumulative hip contact stress predicts osteoarthritis in DDH. Clin Orthop Relat Res 466: 884-891, 2008.

25. Offierski CM and Macnab I: Hip-spine syndrome. Spine (Phila Pa 1976) 8: 316-321, 1983.

26. Sasaki K, Hongo M, Miyakoshi N, Matsunaga T, Yamada S, Kijima Hand Shimada Y: Evaluation of sagittal spine-pelvis-lower limb alignment in elderly women with pelvic retroversion while standing and walking using a three-dimensional musculoskeletal modelw. Asian Spine J 11: 562-569, 2017.
27. YoshimotoH,Sato S,Masuda T,Kanno T,Shundo M,Hyakumachi T and Yanagibashi Y: Spinopelvic alignment in patients with osteoarthrosis of the hip: A radiographic comparison to patients with low back pain. Spine (Phila Pa 1976) 30: 1650-1657, 2005.

This work is licensed under a Creative Commons Attribution-NonCommercial-NoDerivatives 4.0 International (CC BY-NC-ND 4.0) License. 